\title{
Piomiositis primaria del músculo esternocleidomastoideo: Reporte de un caso y revisión de la literatura
}

\author{
Primary pyomyositis of the sternocleidomastoid muscle: \\ A case report and review of the literature
}

Claudio Carnevale S1, Diego Arancibia T11, Guillermo Til P1, Pedro Sarría E1, Manuel Tomás B1'.

\begin{abstract}
RESUMEN
La piomiositis es una infección bacteriana del músculo estriado, siendo extremadamente rara la afectación de la musculatura cervical. Se ha asociado en nuestro medio a enfermedades crónicas como la diabetes y a la inmunodepresión. Presentamos a un paciente de 67 años que acude al servicio de urgencias por tumoración laterocervical de rápido crecimiento, negando antecedentes de interés a excepción de diabetes mellitus tipo II. Se le realizó estudio de imagen con tomografía computarizada con contraste, observándose aumento de volumen del músculo esternocleidomastoideo izquierdo y se empezó tratamiento empírico con antibióticos endovenosos. Dada la evolución tórpida finalmente se realizó drenaje quirúrgico bajo anestesia general con mejoría de los parámetros clínicos y analíticos. La piomiositis de los músculos cervicales es muy rara (0,4\%-1\% de todos los casos) siendo el esternocleidomastoideo el músculo del cueIlo más frecuentemente afectado. La tomografía computarizada representa la prueba de imagen de elección, permitiendo un rápido diagnóstico llegando a poder diferenciar este cuadro de otros similares. Considerando que muchos de estos pacientes presentan comorbilidades asociadas y/o inmunosupresión, es de extrema importancia diagnosticarlos precozmente y empezar un tratamiento adecuado que dependerá del grado y extensión de la infección. A pesar de ser una entidad poco frecuente, su incidencia está en aumento en nuestro medio asociada a la infección por VIH y otras condiciones de inmunodepresión. Hay que tenerla en mente en el diagnóstico diferencial de las tumoraciones laterocervicales porque solo con una alta sospecha clínica se podrá llevar al cabo un diagnóstico precoz y un tratamiento adecuado.
\end{abstract}

Palabras clave: Piomiositis; músculo esternocleidomastoideo; drenaje quirúrgico; inmunodepresión.

\section{ABSTRACT}

Pyomyositis is a bacterial infection of the striated muscle that may affect the cervical musculature in very few cases. In the occidental world it has been associated with chro-

1 Médico del Servicio de Otorrinolaringología y Cirugía de Cabeza y Cuello, Hospital Universitari Son Espases. Palma de Mallorca, Islas Baleares, España.

Recibido el 19 de enero, 2018. Aceptado el 18 de junio, 2018. 
nic diseases as diabetes and immunosuppression. We present a 67 years old patient attended to the Emergency Department because of a laterocervical fast growth tumor, without an interesting clinical history with the exception of type II diabetes mellitus. A CT scan with contrast showed an increased volume in the left sternocleidomastoid muscle, so an empiric treatment with intravenous antibiotics was started. Because of a bad evolution we finally performed a surgical drainage of the abscess under general anesthesia with an improvement of symptoms and laboratory markers. The pyomyositis of cervical muscles is very rare $(0.4-1 \%$ of all cases) and the sternocleidomastoid muscle is the most commonly affected cervical muscle. CT scan is the gold standard imaging technique, because it allows to diagnose this disease and rule out other similar entities. If we consider that many patients present with associated comorbidities and/ or immunosuppression, it's very important to perform a rapid diagnosis and to begin a correct treatment that depends on the grade and extension of the infection. Although polymyositis of the sternocleidomastoid muscle is rare, its incidence is increasing associated to HIV infection and other immunosuppressive conditions. We have to keep in mind this pathology in the differential diagnosis of laterocervical tumors because high clinical suspicion is necessary to make a rapid diagnosis and a correct treatment.

Key words: Pyomyositis; sternocleidomastoid muscle; surgical drainage; immunosuppression.

\section{INTRODUCCIÓN}

La piomiositis se define como una infección bacteriana del músculo estriado, con una incidencia predominante en las regiones de latitudes bajas, por lo que suele clasificarse como tropical o no tropical, aunque la incidencia en regiones no tropicales ha aumentado en las últimas décadas asociado a pacientes inmunosuprimidos ${ }^{1}$. La incidencia en nuestro medio es de 1 caso por 2.000 personas, afectando principalmente a pacientes inmunodeprimidos, VIH positivos 0 con enfermedades crónicas como la diabetes mellitus ${ }^{2}, 3$. Se puede tratar de una infección primaria o secundaria a procesos que afectan a los tejidos alrededor del músculo. Aunque puede afectar prácticamente a cualquier músculo estriado del cuerpo, suele comprometer más frecuentemente la musculatura de las extremidades inferiores (cuádriceps, bíceps, psoas), siendo excepcional la afectación de los músculos cervicales (0,5\%-4\%). El objetivo de este artículo es presentar el caso de un paciente con piomiositis primaria del músculo esternocleidomastoideo y realizar una revisión de la literatura publicada sobre el tema. A pesar de que la piomiositis de la musculatura cervical representa una entidad rara, hay que tenerla en cuenta en el diagnóstico diferencial de las tumoraciones cervicales en urgencias, considerando el aumento de su incidencia en nuestro medio y el hecho de que se asocia sobre todo a pacientes con cuadros de inmunodepresión, donde la evolución de la enfermedad puede ser desfavorable si no se realiza un diagnóstico rápido y un tratamiento adecuado.

\section{CASO CLÍNICO}

Se trata de un paciente de sexo masculino de 67 años de edad, con antecedente de diabetes mellitus tipo 2, hipertensión arterial y dislipidemia, no fumador, que acudió al servicio de urgencias de nuestro hospital por aparición de una tumoración laterocervical izquierda dolorosa a la palpación y de rápido crecimiento. A la anamnesis el paciente negaba procesos infecciosos en las semanas previas, así como traumatismos en la región cervical. Al examen físico, el paciente se encontraba afebril, con buen estado general y signos vitales normales, siendo el único hallazgo de interés un aumento de partes blandas a nivel laterocervical izquierdo, palpándose a nivel del tercio superior y medio del músculo esternocleidomastoideo (ECM) una tumoración de $6 \times 4 \mathrm{~cm}$ en sus ejes longitudinal y transversal, de consistencia dura, con escaso eritema del tejido cutáneo. A la exploración con 
videoendoscopio se apreciaba la obliteración del seno piriforme izquierdo, con retención de saliva y sin ninguna lesión macróscopica identificable. Las pruebas de laboratorio evidenciaban una leucocitosis de $33.800 / \mu \mathrm{L}$ con neutrofilia $(91,8 \%)$ y proteína $\mathrm{C}$ reactiva de $25 \mathrm{mg} / \mathrm{dL}$. Ante los hallazgos descritos se realizó una tomografía computarizada de cuello con contraste donde se apreció un aumento de tamaño del músculo ECM izquierdo con leve edema subcutáneo asociado (Figura 1). Debido a los hallazgos clínicos, imagenológicos y de laboratorio que apuntaban a un cuadro infeccioso, se decidió iniciar antibioticoterapia endovenosa de amplio espectro con cefotaxima $1 \mathrm{~g}$ cada 8 horas y clindamicina $600 \mathrm{mg}$ cada 6 horas. Ante una evolución tórpida en las siguientes 48 horas, se realizó una nueva tomografía computarizada con contraste, mostrando una imagen compatible con miositis del ECM y presencia de colección a nivel de su margen anterior (Figura 2). Se realizó drenaje quirúrgico bajo anestesia general, biopsia de tejido muscular y se envió una muestra del líquido drenado para el estudio microbiológico. El paciente presentó una evolución adecuada, con mejoría clínica y normalización de los parámetros analíticos. Al cuarto día posoperatorio se procedió al alta hospitalaria y al seguimiento ambulatorio, confirmándose buena evolución en los 3 meses posteriores. El informe de anatomía patológica

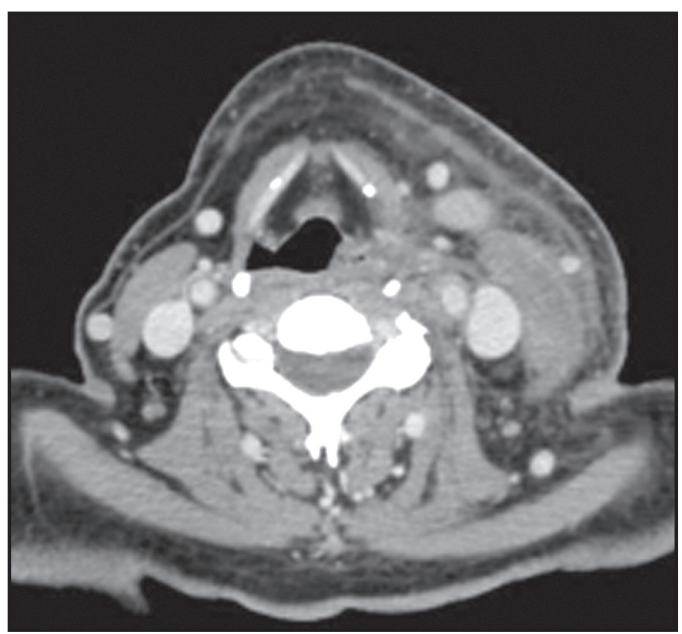

Figura 1. TC con contraste: aumento de volumen del músculo esternocleidomastoideo izquierdo. describió características histopatológicas compatibles con piomiositis del músculo ECM.

\section{DISCUSIÓN}

La piomiositis es una infección bacteriana del músculo estriado, con una incidencia predominante en regiones tropicales, aunque la incidencia en regiones no tropicales ha aumentado en las últimas décadas siendo de 1 caso por 2.000 personas y afectando principalmente a pacientes inmunodeprimidos, VIH positivos 0 con enfermedades crónicas como la diabetes mellitus $^{1-3}$. Puede ser una infección primaria 0 secundaria a procesos que afectan a los tejidos alrededor del músculo. Se distinguen dos formas principales de piomiositis: la variante tropical y la no tropical. En la primera se describen dos picos de incidencia entre 2 a 5 años y entre 35 a 40 años de edad, con una relación hombre/mujer de $4: 1$, mientras que en la segunda variante los picos de incidencia son entre 30 a 50 años y entre 60 a 70 años de edad, con relación entre hombre/ mujer de 3:1. En la forma no tropical, generalmente se trata de pacientes con comorbilidades, diabetes mellitus, neoplasias, enfermedades reumatológicas, inmunosupresión, mientras que la forma tropical suele ocurrir en individuos

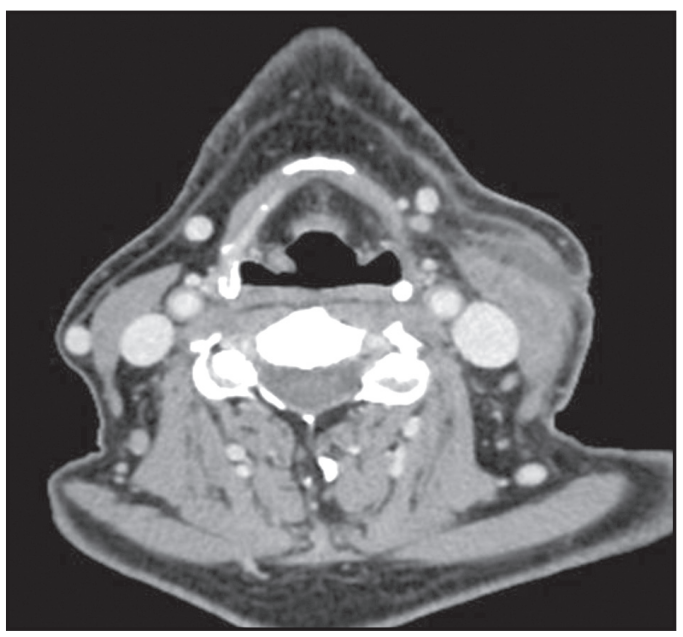

Figura 2. TC con contraste: colección a nivel del borde anterior del esternocleidomastoideo izquierdo. 
previamente sanos. En términos generales, el trauma se asocia solo en $5 \%$, aunque parece tomar mayor importancia en la forma no tropical. Aunque puede afectar prácticamente a cualquier músculo estriado del cuerpo, interesa más frecuentemente la musculatura de las extremidades inferiores $(26 \%)$, seguidas por las superiores (14\%), siendo el cuádriceps femoral el músculo más comúnmente afectado. Los músculos del cuello solo representan el $0,4 \%$ de los casos, siendo el más afectado el ECM y junto con la cabeza $(0,4 \%)$ y la mano $(0,1 \%)$ son los sitios de menor incidencia de piomiositis primaria. Lo más frecuente es el compromiso de un solo músculo, pero a pesar de eso, está descrita la afectación concomitante de varios músculos entre el $11 \%$ y el $43 \%$ de los casos.

En el $98 \%$ de los casos el origen es bacteriano, donde destaca como primer agente infeccioso el Staphylococcus aureus, responsable hasta del $90 \%$ de los casos. El $S$. aureus meticilino resistente es frecuente (hasta el $25 \%$ ) en la forma no tropical de pacientes diabéticos en Estados Unidos. Otras bacterias implicadas son el Streptococcus (forma no tropical, donde destaca el beta-hemolítico del grupo A) y los bacilos gram-negativos, principalmente Escherichia coli y salmonella. Los agentes fúngicos (Candida albicans) son poco frecuentes, aunque con tendencia a ser más elevados en la forma no tropical ( $2 \%)$.

La historia natural de la piomiositis fue descrita por Chiedozi en 1979, quien reportó una cohorte de 205 casos. En su descripción, refiere tres estadios de la enfermedad. El estadio 10 etapa "invasiva", que ocurre en los primeros 10 días y en la que se encuentra el $2 \%$ de los pacientes, es la fase inicial de la enfermedad, con poca sintomatología y escasa respuesta inflamatoria sistémica. El estadio 20 etapa "supurativa", entre los 10 y 20 días de evolución, es la etapa en que más frecuentemente se encuentran los pacientes al momento de la consulta $(90 \%)$. La inflamación local, el dolor y la tumefacción aumentan, la fiebre y la leucocitosis son más marcadas, siendo el absceso evidente a la exploración. Finalmente, el estadio 3 o etapa "tardía", es la etapa más avanzada, en la que se presenta el $5 \%$ de los pacientes y se presenta con un cuadro séptico.

Los exámenes de laboratorio muestran parámetros inflamatorios inespecíficos: es frecuente observar leucocitosis con neutrofilia y elevación de la velocidad de sedimentación globular. Los cultivos microbiológicos suelen ser negativos en $60 \%-80 \%$ de los casos. En las formas tropicales podemos encontrar eosinofilia. En el caso presentado observamos una leucocitosis de $33.800 / \mu \mathrm{l}$ con desviación a la izquierda, cultivo microbiológico del líquido drenado negativo y biopsia del tejido muscular compatible con absceso y reacción de cuerpo extraño. Entre las pruebas de imagen, la técnica de elección es la tomografía computarizada con contraste, muy útil en el diagnóstico diferencial de los procesos infecciosos/tumorales de la región cervicofacial, en el manejo inicial de los pacientes y en el seguimiento. La ecografía puede ser utilizada para realizar punciones ecoguiadas de las colecciones purulentas. Nosotros consideramos necesaria la realización urgente de una tomografía computarizada con contraste que permite realizar un diagnóstico precoz y nos guía en la elección del tratamiento más adecuado. Considerando que la mayoría de los pacientes presentan comorbilidades crónicas y/o cuadros de inmunosupresión, se entiende que un retraso en el diagnóstico con la aparición de complicaciones puede ser fatal. Con respecto al tratamiento, depende del estadio de la infección y del compromiso sistémico. En las fases iniciales, suele ser suficiente el tratamiento antibiótico por vía endovenosa durante al menos 7-10 días seguido de otras 5-7 semanas de tratamiento oral ambulatorio, teniendo en cuenta, a la hora de elegir el antibiótico, la alta prevalencia del $S$. aureus como agente causal más frecuente. La asociación de una cefalosporina de $3^{a}$ generación con clindamicina hasta el resultado del antibiograma es considerado el tratamiento empírico de elección. Con la progresión del proceso y la formación de un absceso, se establece la necesidad de asociar un drenaje quirúrgico, incluso eliminando porciones de tejido muscular inviable ${ }^{1}$. En estos casos suele ser útil la colocación de drenajes tipo Penrose y la realización de lavados con antibiótico a través de ellos en los días sucesivos a la cirugía.

\section{CONCLUSIÓN}

La piomiositis es una infección rara, siendo excepcional a nivel cervical donde el músculo más 
comúnmente afectado es el esternocleidomastoideo. Los pilares del tratamiento son la antibioticoterapia endovenosa asociada a drenaje quirúrgico según la fase clínica específica. En nuestro medio suele tratarse de pacientes con enfermedades crónicas y/o inmunodepresión por lo que la alta sospecha clínica y el diagnóstico

\section{BIBLIOGRAFÍA}

1. Crum NF. Bacterial pyomyositis in the United States. Am J Med 2004; 117: 420-8.

2. Martín-Millán M, García-Ibarbia C, GutiérrezCuadra M, Gutiérrez-Santiago M, FernándezSampedro M, González-Macías J, et al. Piomiositis: una revisión retrospectiva en un hospital terciario del norte de España. Enferm Infecc Microbiol Clin 2006; 24: 173-7.

3. Patel SR, Olenginski tP, Perruquet Jl, Harrington TM. Pyomyositis: clinical features and predisposing conditions. J Rheumatol 1997; 24: $1734-8$.

4. Bickels J, Ben-Sira L, Kessler A, Wientroub S. Primary Pyomyositis. J Bone Joint Surg Am 2002; 84: 2277-86.

5. Chiedozi LC. Pyomyositis. Review of 205 cases in 112 patients. Am J Surg 1979; 137: 255-9. precoz son determinantes para evitar la progresión de la enfermedad hacia cuadros sistémicos más graves. La tomografía computarizada con contraste representa sin duda la técnica de imagen de elección, permitiendo un diagnóstico precoz así como el seguimiento de los pacientes en caso de evolución tórpida.

6. Gómez-Reino JJ, Aznar JJ, Pablos Jl, Díaz-González F, LAfFon A. Nontropical pyomyositis in adults. Semin Arthritis Rheum 1994; 23: 396-405.

7. Hall RL, Callaghan JJ, Moloney e, Martínez S, Harrelson JM. Pyomyositis in a temperate climate. Presentation, diagnosis, and treatment. J Bone Joint Surg Am 1990; 72: 1240-4.

8. Zalavras CG, Rigopoulos N, Poultsides L, Patzakis MJ. Increased oxacillin resistance in thigh pyomyositis in diabetic patients. Clin Orthop Relat Res 2008; 466: 1405-9.

9. Flier S, Dolgin SE, Saphir RL, Shlasko E, Midulla P. A case confirming the progressive stages of pyomyositis. J Pediatr Surg 2003; 38: 1551-3.

10. Mueller PR, Ferrucci JT Jr, Wittenberg J, Simeone JF, Butch RJ. lliopsoas abscess: treatment by CT-guided percutaneous catheter drainage. AJR Am J Roentgenol 1984; 142: 359-62.

Dirección: Claudio Carnevale

Servicio de Otorrinolaringología y Cirugía de Cabeza y Cuello, Hospital Universitari Son Espases.

Palma de Mallorca, Islas Baleares, España

E mail: claudio.carnevale.orl@gmail.com 